\title{
Hausstaubmilbenallergie - SLIT-Präparat jetzt auch für Kinder zugelassen
}

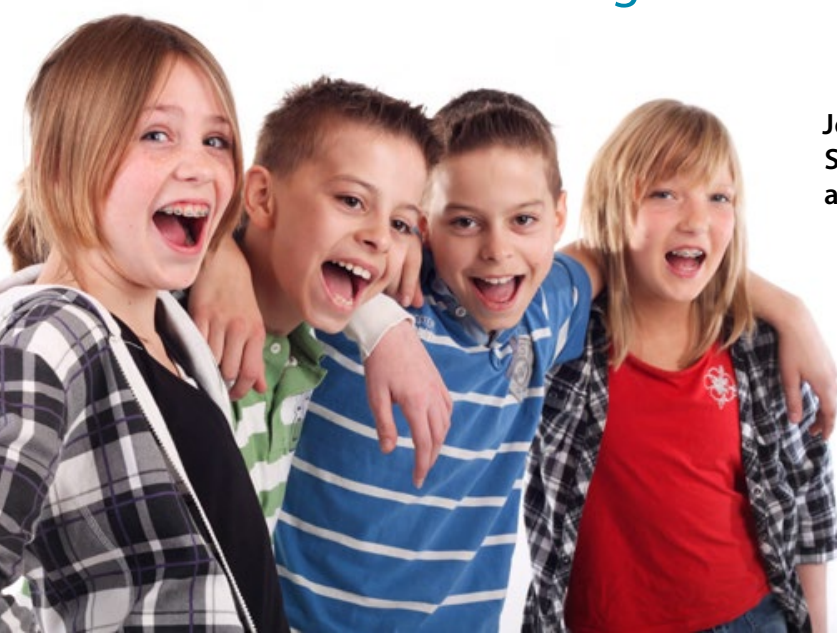

\begin{abstract}
Jetzt nicht mehr nur für Erwachsene: SLIT-Präparat für Hausstaubmilbenallergiker ab 12 Jahren zugelassen.
\end{abstract}

bekämpfen. Die erweiterte Zulassung deckt nun auch Patienten im Alter von zwölf bis 17 Jahren mit moderater bis schwerer hausstaubmilbeninduzierter allergischer Rhinitis ab, die trotz der Verwendung symptomlindernder Medikamente Beschwerden haben. Die Zulassung basiert auf Daten des globalen klinischen Ent-

as Paul-Ehrlich-Institut hat die Zulassung der sublingualen Immuntherapie (SLIT) ACARIZAX ${ }^{\circledR}$ gegen Hausstaubmilbenallergie auf Kinder und Jugendliche ab zwölf Jahren erweitert. ACARIZAX ${ }^{\circledR}$ ist seit 2016 in Deutschland verfügbar und war bisher nur für Erwachsene zugelassen, um die Ursache einer hausstaubmilbeninduzierten allergischen Atemwegserkrankung in den oberen und unteren Atemwegen zu wicklungsprogramms von ACARIZAX ${ }^{\circledR}$, die das günstige Wirksamkeits-, Sicherheitsund Verträglichkeitsprofil der SLIT-Tablette bestätigen und den Ergebnissen früherer Studien mit Erwachsenen entsprechen. "Mit der neuen Indikation für ACARIZAX ${ }^{\circledR}$ können Jugendliche mit allergischer Rhinitis frühzeitig behandelt werden", so Prof. Dr. Eike Wüstenberg, medizinischer Leiter von ALK Deutschland. ALK geht davon aus, dass Kinder und Jugendliche im Alter zwi- schen zwölf und 17 Jahren etwa 20\% der Hausstaubmilbenallergiker ausmachen. Eine frühzeitige Behandlung, welche die Ursache der Krankheit bekämpft, ist bei Hausstaubmilbenallergikern besonders wichtig, da ihre allergische Rhinitis häufig progressiv verläuft, und diese Patienten ein dreifach erhöhtes Risiko haben, an Asthma zu erkranken [Shaaban R er al. Lancet 2008;372: 1049-57].

ACARIZAX ${ }^{\circledast}$ ist die erste HausstaubmilbenSLIT, die in Europa zugelassen ist und zudem das erste Präparat für eine spezifische Immuntherapie (SIT) überhaupt, das zur Therapie eines nicht gut kontrollierten allergischen Asthmas von Erwachsenen zugelassen ist, wenn gleichzeitig eine allergische Rhinitis vorliegt. Hinter der Zulassung von ACARIZAX ${ }^{\circledR}$ steht das weltweit größte klinische SIT-Entwicklungsprogramm mit mehr als 6.000 Patienten auf drei Kontinenten. Die gute Studienlage von ACARIZAX ${ }^{\circledR}$ führte im Februar 2017 dazu, dass das Wissenschaftskomitee der Global Initiative for Asthma (GINA) in ihrem Bericht für 2017 erstmalig die SIT als Behandlungsoption bei hausstaubmilbenbedingtem Asthma empfiehlt.

Nach Informationen von ALK

\section{Schweres Asthma: Patientenumfrage zeigt erhöhten Handlungsbedarf}

Dis ie Daten der Patientenumfrage "Still Fighting for Breath" zeigen, dass Asthma in Europa trotz der weitverbreiteten Verfügbarkeit inhalativer Medikamente eine enorme Belastung für das Privat- und Berufsleben der Betroffenen darstellt [Katsaounou $\mathrm{P}$ et al. \#858 presented at EACCI Congress 2017]. Die Umfrage offenbarte, dass es unter Erwachsenen und Kindern mit schwerem Asthma nach wie vor einen hohen Anteil an Patienten gibt, bei denen die Krankheit nicht unter Kontrolle ist $(94 \%)$. Besonders hervorzuheben ist, dass in der Auswertung der neuen Daten eine signifikante Diskrepanz zwischen der "wahrgenommenen" und der "realen" Asthmakontrolle gefunden wurde. So gaben $46 \%$ der befragten Patienten in ihrer Selbsteinschätzung an, ihre Symptome seien „unter Kontrolle“. Diese Angabe steht im Gegensatz zu den $6 \%$ der Patienten, die gemäß den klinischen Leitlinien der Global Initiative for Asthma tatsächlich als "kontrolliert" eingestuft wurden.

Eine erfolgreiche Asthmakontrolle ist essenziell, damit Patienten mit schwerem Asthma optimal medizinisch versorgt sind und ihre Lebensqualität erhalten bleibt. Von einer Behandlung mit Biologika wie dem AntiIgE-Antikörper Omalizumab (Xolair ${ }^{\circledR}$ ), können geeignete Patienten erheblich profitieren, wie zahlreiche Studien mittlerweile zeigen konnten. So können die Ergebnisse einer deutschen Beobachtungsstudie, in der 134 Ärzte 280 Patienten neu auf das Anti-IgE eingestellt hatten, belegen, dass sich mit Omalizumab die Exazerbationsrate deutlich senken lässt. Nach einem Behand- lungszeitraum von sechs Monaten war die Exazerbationsrate um $82 \%$ gesunken, die der Hospitalisierungen um $78 \%$. [Korn $\mathrm{S}$ et al. Respir Med 2009; 103: 1725-31].

Darüber hinaus mehren sich die Studien, die darauf hindeuten, dass Omalizumab auch bei Infekt-Exazerbationen wirken könnte. Die PROSE-Studie, in der asthmakranke Schulkinder zwei Monate vor Ferienende entweder Omalizumab oder eine Verdopplung ihrer Dosis an inhalativen Glukokortikoiden erhalten hatten, sollte die mit dem Schulbeginn einsetzende Exazerbations-„„Epidemie“" verhindern [Teach SJ et al. J Allergy Clin Immunol 2015; 136: 147685]. Dies gelang bei Patienten mit einer Exazerbation in der Run-in-Phase nur in der Omalizumab-Gruppe: Hier blieb der sonst übliche Anstieg der Exazerbationsrate praktisch völlig aus.

Nach Informationen von Novartis 